\title{
Overnight oximetry in children undergoing adenotonsillectomy: a single center experience
}

\author{
C. Carrie Liu', Kathleen H. Chaput ${ }^{2}$, Valerie Kirk ${ }^{3}$ and Warren Yunker ${ }^{1 *}$
}

\begin{abstract}
Background: Obstructive sleep apnea (OSA) is the most common indication for adenotonsillectomy in children. Home-based sleep oximetry continues to be used in the diagnosis of pediatric OSA despite a lack of correlation with lab-based polysomnography. This study investigates whether factors influence surgeons in selecting patients for home-based sleep oximetry, how the study findings are used in patient management, and whether abnormal oximetry findings are associated with post-operative complications.
\end{abstract}

Methods: A retrospective review was performed on children with suspected OSA who had undergone a tonsillectomy and/or an adenoidectomy over a three-year period. Demographic features, comorbidities, preoperative oximetry results, and post-operative complications were recorded. Data analysis consisting primarily of logistic regression was performed using Stata 12.0 (College Station, Texas).

Results: Data was collected from 389 children. Two hundred and seventy-one children underwent pre-operative oximetry (69.7\%). There was no significant association between age or the presence of comorbidities and the likelihood of undergoing pre-operative sleep oximetry. The post-operative complication rate was $0.8 \%$. There was no significant association between abnormal sleep oximetry parameters and post-operative complications. Children with one or more abnormal sleep oximetry parameters were more likely to be observed in hospital for at least one night (OR 2.4, $p<0.0001)$.

Conclusions: Our study suggests that surgeons are using home-based sleep oximetry findings to inform the postoperative care of children with suspected OSA, as those with abnormal home-based sleep oximetry findings were more likely to be observed in hospital. These hospital admissions may be unnecessary given the poor correlation of home-based oximetry and PSG as well as the low rate of serious post-operative complications.

Keywords: Sleep oximetry, Obstructive sleep apnea, Sleep disordered breathing

\section{Introduction}

Obstructive sleep apnea (OSA) has a prevalence of up to $10 \%$ in the pediatric population [1] and can result in neurocognitive and cardiovascular sequelae [2-5]. Polysomnography (PSG) is the gold standard for diagnosing OSA [6]. Despite their diagnostic value, lab-based polysomnography can be difficult to access [7]. A recent

\footnotetext{
* Correspondence: warren.yunker@albertahealthservices.ca

${ }^{1}$ Sections of Otolaryngology - Head and Neck Surgery and Pediatric Surgery, Department of Surgery, Cumming School of Medicine, University of Calgary, Calgary, Alberta, Canada

Full list of author information is available at the end of the article
}

survey of the Canadian Pediatric Sleep Network showed significant geographical variance in the availability of PSG, with wait times ranging from less than 1 month to 2 years [8]. Furthermore, only $12 \%$ of children in Canada undergo polysomnography prior to adenotonsillectomy. The lack of accessibility to PSG has motivated health care providers to seek and investigate portable diagnostic means [8].

Lab-based overnight oximetry has been shown to have satisfactory correlation with PSG in the diagnosis of moderate to severe OSA [9]. The sensitivity and specificity of home-based overnight oximetry, however, is 67 and $60 \%$, respectively, in the diagnosis of children with 
moderate OSA [10]. In contrast to lab-based oximetry, the interpretive internal algorithms that generate homebased oximetry reports are computerized and not manually validated, resulting in the potential incorporation of artifact into the final reports. Due to the poor agreement between home-based oximetry and PSG, the current recommendations do not support the use of this modality in the diagnosis of pediatric OSA [11].

Notwithstanding, our institution has observed the ongoing use of home-based sleep oximetry, both in public and private care settings. A review of the recent literature suggests that many clinicians are still using this modality as part of their standard approach to children referred for OSA [12-15]. In a survey of National Health Services otolaryngology departments in the UK, $84 \%$ of respondents reported using pre-operative pulse oximetry and among these practitioners, 58\% used home-based sleep oximetry [15]. The continued use of home-based sleep oximetry likely reflects the lack of access to PSG that is seen in many care settings, predominately in publicly funded health systems. It may, however, also reflect a lack of awareness regarding current recommendations.

Given the continued use of home-based sleep oximetry in many institutions, it is important to investigate how this modality is being used and its effects on patient care. The current study investigates the experience with home-based overnight sleep oximetry at our institution. Specifically, we investigated the factors that influence practitioners in ordering home-based oximetry. We also examined whether sleep oximetry findings affect patient management in terms of overnight admission versus day surgery. Finally, we investigated whether specific abnormal oximetry findings are associated with peri-operative complications.

\section{Methods}

This was a retrospective review of children who underwent tonsillectomy and/or adenoidectomy surgery at the Alberta Children's Hospital during the period of January 1, 2005 to December 31, 2009. Approval for the study was obtained from the Conjoint Health Research Ethics Board at the University of Calgary.

\section{Patient selection}

The study population consists of a series of children undergoing adenotonsillectomy, tonsillectomy, or adenoidectomy surgery at the Alberta Children's Hospital. Subjects were identified using a computerized search and were included if the following criteria were met: children aged 1-18 years who underwent adenotonsillectomy, adenoidectomy, or tonsillectomy, and with a suspected diagnosis of SDB/OSA. Children were excluded if they had a history of prior airway surgery (i.e. cleft palate repair, uvulopalatoplasty, adenoidectomy and/or tonsillectomy).

\section{Data collection}

Hospital charts were reviewed and pertinent data regarding demographic information, comorbidities, pre-operative oximetry results, surgery performed, and peri-operative complications were recorded. Serious complications included death, cardiorespiratory arrest, acute respiratory distress, and unplanned pediatric intensive care admissions. The length of hospitalization was recorded. At our institution, children are admitted after adenotonsillectomy if they are younger than 3 years of age, have severe OSA as defined by $\mathrm{AHI} \geq 10$ or oxygen saturation nadir $<80 \%$, or if they have specific comorbid conditions (ie. trisomy 21, neuromuscular disease, craniofacial abnormalities) [16]. Children may also be admitted if there are post-operative concerns such as poor oral intake, supplemental oxygen requirements, or uncontrolled pain. An unnecessary admission is defined as an admission outside of these parameters.

Oximetry data generated by pre-operative home studies were recorded. All home-based oximetry studies were performed using the N595 Nellcor oximeter (Medtronic). The averaging time was set at the fast mode (2-3 s). As defined by Nixon et al., a "desaturation" was defined as a decrease in oxygen saturation of $\geq 4 \%$ and a "cluster of desaturations" was scored as $\geq 5$ desaturations occurring in a 10- to 30-min period [17]. Mean and minimum oxygen saturation, time spent below $90 \%$ and time spent below $80 \%$ was also recorded.

There are validated scoring systems for lab-based sleep oximetry that record the number of desaturations below a certain percentage [17]. The respirology team at our institution chose a modified scoring system to interpret and classify overnight oximetry results. The McGill scoring system was based on data obtained using an N200 oximetry unit [17]. This unit allows for the visualization of the pulse waveform, which a measure of signal integrity. The oximetry devices at the Alberta Children's Hospital do not collect pulse waveform data. As such, we used a three-scale system that blended the McGill classifications of two and three, with the intention of avoiding overinterpretation or under-interpretation of the oximetry findings. The oximetry outcome groups were 1 normal (no desaturations), 2 - abnormal (desaturations which did not meet criteria for diagnosis of OSA), 3 findings consistent with OSA (clusters of desaturations occurring regularly throughout the recording).

\section{Data analysis}

Demographic data was summarized using descriptive statistics. Logistic regression was performed to examine the factors that contribute to ordering oximetry, the association between abnormal oximetry findings and complications rates, and whether abnormal oximetry findings were associated with an increased length of stay 
Table 1 Study subject characteristics

\begin{tabular}{lllll}
\hline & $\begin{array}{l}\text { Total } \\
(n=389)\end{array}$ & $\begin{array}{l}\text { Adenotonsillectomy } \\
(n=271)\end{array}$ & $\begin{array}{l}\text { Adenoidectomy } \\
(n=95)\end{array}$ & $\begin{array}{l}\text { Tonsillectomy } \\
(n=23)\end{array}$ \\
\hline Age in years (SD) & $5.8(3.3)$ & $6.0(3.2)$ & $4.7(2.8)$ & $8.7(4.6)$ \\
Males (\%) & $214(55 \%)$ & $148(55 \%)$ & $54(57 \%)$ & $12(52 \%)$ \\
Pre-op oximetry performed (\%) & $271(70 \%)$ & $191(70 \%)$ & $64(67 \%)$ & $16(70 \%)$ \\
\hline
\end{tabular}

in hospital. All analyses were performed using STATA 12.0 (College Station, Texas).

\section{Results}

A total of 389 children were included. Table 1 summarizes the demographic information of the study subjects. In total, home-based overnight sleep oximetry was performed in 271 (69.7\%) children with suspected OSA/ SDB undergoing surgery. Thirty children $(7.7 \%)$ had a chromosomal abnormality, metabolic syndrome, or cardiac condition. There was no association between age or comorbidities and the likelihood of undergoing preoperative sleep oximetry (OR 1.0, $p>0.05$ and $0.9, \mathrm{p}>$ 0.05 , respectively).

Of 389 operations, there were a total of $3(0.8 \%)$ serious complications, with 2 resulting in intensive care unit (ICU) admissions (Tables 2 and 3). One child had post-obstructive desaturations secondary to aspiration pneumonia, which improved with conservative management with intravenous antibiotics and incentive spirometry on the inpatient ward. The child was discharged home after a 5-day admission and was deemed by the pediatrics inpatient team to not require outpatient followup from a pulmonary perspective. The other two children had significant obstructive events post-operatively, with prolonged apneic spells and oxygen desaturations to below 70\%. Both children required admission to the ICU for administration of oxygen via face mask. They were discharged 3 and 7 days later with community follow-up with a pediatrician. We observed no significant association between abnormal sleep oximetry parameters and postoperative complications (Table 4).

Two hundred and nineteen children (56.3\%) were observed in hospital for at least one night, while the remainder had same-day discharge. Of those with admissions, 57 were admitted for age under 3 , a comorbidity that warranted admission, or an oxygen saturation nadir of $<80 \%$. One $(0.5 \%)$ child was admitted for post-operative fevers.

Table 2 Serious post-operative complications

\begin{tabular}{llll}
\hline Patient & Age (years) & Comorbidities & Surgery performed \\
\hline 1 & 8 & No & AT \\
2 & 2 & No & AT \\
3 & 1 & No & A \\
\hline
\end{tabular}

$A T$ adenotonsillectomy, $A$ adenoidectomy
The remaining 162 admissions did not have clear indications and are considered unnecessary.

One hundred and ninety-one children (49.1\%) had one or more abnormal oximetry parameter (Table 5). When controlled for age, those with at least one abnormal sleep oximetry parameter had a 2.4 increase in the odds of having an unnecessary admission, compared to children with normal parameters $(p<0.0001)$. Specifically, children who had oxygen nadirs $<90 \%$ and clusters of desaturations on oximetry had 2.4 and 2.1 times the odds of unnecessary admissions, respectively ( $\mathrm{p}<0.0001$ for both). The proportion of children in each oximetry outcome group who had an unnecessary admission is shown in Table 6.

\section{Discussion}

Currently, the use of home-based studies is not recommended in the diagnosis of pediatric OSA [11]; however, there remains utilization of home-based sleep oximetry, possibility as a result of the resource scarcity that is seen in many care settings $[14,15]$. Our study showed that there is variability with which home-based sleep oximetry is ordered and interpreted among surgeons. In our cohort, 30.3\% of children with suspected OSA/SDB did not undergo pre-operative oximetry. This likely reflects practice variation among surgeons; some surgeons diagnose and treat OSA on the basis of the clinical history and physical exam, whereby others defer diagnosis and treatment until after the overnight sleep oximetry study is performed. Surgeons may also be using sleep oximetry as a means to triage and determine the urgency of surgery. Finally, our study suggests that surgeons may be using oximetry to inform perioperative patient management, as those with abnormal oximetry findings were more likely to be observed in hospital overnight.

The association between abnormal oximetry findings and increased post-operative observation suggests that

Table 3 Serious post-operative complications and the corresponding oximetry findings

\begin{tabular}{|c|c|c|c|c|c|}
\hline Patient & Mean $\mathrm{SpO}_{2}$ & Min $\mathrm{SpO}_{2}$ & $\begin{array}{l}\% \text { time with } \\
\mathrm{SpO}_{2}<90 \%\end{array}$ & $\begin{array}{l}\% \text { time with } \\
\mathrm{SpO}_{2}<80 \%\end{array}$ & Clusters \\
\hline 1 & $96 \%$ & $89 \%$ & $1 \%$ & $0 \%$ & No \\
\hline 2 & $88 \%$ & $58 \%$ & $63 \%$ & $2 \%$ & Yes \\
\hline 3 & $96 \%$ & $76 \%$ & $2 \%$ & $0.1 \%$ & Yes \\
\hline
\end{tabular}

Min $\mathrm{SpO}_{2}$, minimum oxygen saturation; clusters defined by $\geq 5$ desaturation $\geq 4 \%$ in a $10-30$-min period 
Table 4 Complications and abnormal oximetry parameters

\begin{tabular}{lll}
\hline Parameter & Odds ratio $(95 \% \mathrm{Cl})$ & $p$-value \\
\hline Nadir $<90 \%$ & $1.0(0.1,11.4)$ & 0.9 \\
Nadir $<80 \%$ & $7.0(0.6,77.9)$ & 0.1 \\
$\mathrm{SpO}_{2}<90 \% \geq 1 \mathrm{~min}$ & $3.5(0.3,38.7)$ & 0.3 \\
$\mathrm{SpO}_{2}<80 \% \geq 1 \mathrm{~min}$ & $8.3(0.7,94.8)$ & 0.2 \\
Clusters of desaturations & $3.6(0.3,39.9)$ & 0.3 \\
$\geq 1$ of the above parameters & $2.1(0.2,23.2)$ & 0.6 \\
\hline
\end{tabular}

physicians are using oximetry as a way to estimate the risk of post-operative complications. Overall, we found a very low rate of severe post-operative complications $(0.8 \%)$. This is consistent with the complication rates reported in the literature [18]. We did not find a significant association between any abnormal sleep oximetry parameters and the occurrence of post-operative complications.

Our study contributes to the existing literature in that there appears to be no utility in the use of home-based sleep oximetry, especially as a method of predicting postoperative complications. Furthermore, given the finding that children with abnormal sleep oximetry studies have a higher odd of being admitted, along with the low overall rate of post-operative complications, home-based sleep oximetry and the admissions based on oximetry findings may represent an unnecessary cost to the health care system.

The main limitation of this study is the low number of peri-operative serious complications. As such, our study was not sufficiently powered to analyze the association between respiratory complications and certain sleep oximetry findings. Fortunately, modern surgical and anesthetic techniques are associated with low rates of post-adenotonsillectomy complications. Future studies with larger sample sizes and greater numbers of complication outcomes are required. A second and important limitation of our study is that the sleep oximeter used at our center does not allow for the visualization of the pulse waveform. Visualization of the pulse waveform is important as it allows for the determination of signal integrity and distinction of true desaturations. Unfortunately, modern oximetry units have internal algorithms that delete poor data automatically, precluding the clinician's ability to examine the raw data for true desaturations. As a result, we were unable to use a validated scoring system such as the McGill Score for oximetry

Table 5 Abnormal home-based sleep oximetry results

\begin{tabular}{ll}
\hline Abnormality & No. of children (\%) \\
\hline Nadir $<90 \%$ & $128(33 \%)$ \\
Nadir $<80 \%$ & $88(23 \%)$ \\
$\mathrm{SpO}_{2}<90 \% \geq 1 \mathrm{~min}$ & $143(37 \%)$ \\
$\mathrm{SpO}_{2}<80 \% \geq 1 \mathrm{~min}$ & $23(6 \%)$ \\
Clusters of desaturations & $140(36 \%)$ \\
\hline
\end{tabular}

Table 6 Proportion of children who had unnecessary admissions in each oximetry outcome group

\begin{tabular}{lll}
\hline Oximetry outcome group & $\begin{array}{l}\text { No. of patients } \\
\text { with finding }\end{array}$ & $\begin{array}{l}\text { No. of patients with } \\
\text { admission (\%) }\end{array}$ \\
\hline 1 (normal oximetry) & 198 & $62(31.3 \%)$ \\
2 (desaturations not meeting & 128 & $72(56.3)$ \\
$\begin{array}{l}\text { criteria for OSA) } \\
3 \text { (clusters of desaturations) }\end{array}$ & 140 & $75(53.6 \%)$
\end{tabular}

interpretation [17]. A third limitation of this study is the risk for selection bias given its retrospective design. The study cohort is from a tertiary pediatric hospital, where children who are referred to otolaryngologists for surgery may have been deemed by the referring doctor to have more clinically significant OSA based on the history and physical exam. It is possible that these children were more likely to be admitted based on the clinical history alone. However, our finding is of the association between abnormal sleep oximetry parameters and unnecessary admissions. As there is no known correlation between clinical history and home-based sleep oximetry results, our finding of an increased odds of admission based on abnormal sleep oximetry parameters more likely to reflect the surgeon's interpretation of the oximetry results rather than their evaluation of the patient's clinical history.

\section{Conclusion}

Our study examined the practice patterns surrounding overnight home-based sleep oximetry. We found that surgeons are using sleep oximetry to determine postoperative patient care, as children with abnormal sleep oximetry findings were more likely to be observed in hospital overnight. The ongoing use of home-based sleep oximetry and the hospital admissions that stem from abnormal sleep oximetry findings may represent a waste to the health care system.

\section{Acknowledgements}

None

\section{Authors' contributions}

$\mathrm{CCL}$ was a major contributor in analyzing the data and writing the manuscript, KC was a major contributor in analyzing the data and writing the manuscript, VK was a major contributor in the design of the study and in writing the manuscript, WY was a major contributor in the design of the study and in writing the manuscript. All authors read and approved the final manuscript.

Funding

None

Availability of data and materials

The datasets used and/or analyzed during the current study are available from the corresponding author on reasonable request.

Ethics approval and consent to participate

Approval for the study was obtained from the Conjoint Health Research Ethics Board at the University of Calgary.

Consent for publication Not applicable 


\section{Competing interests}

None

\section{Author details}

'Sections of Otolaryngology - Head and Neck Surgery and Pediatric Surgery, Department of Surgery, Cumming School of Medicine, University of Calgary, Calgary, Alberta, Canada. ${ }^{2}$ Department of Obstetrics and Gynecology and Community Health Sciences, Cumming School of Medicine, University of Calgary, Calgary, Alberta, Canada. ${ }^{3}$ Division of Pediatric Respirology,

Cumming School of Medicine, University of Calgary, Calgary, Alberta, Canada.

Received: 11 June 2019 Accepted: 22 November 2019

Published online: 03 December 2019

\section{References}

1. Lumeng JC, Chervin RD. Epidemiology of pediatric obstructive sleep apnea. Proc Am Thorac Soc. 2008;5(2):242-52.

2. Brockmann PE, Bertrand P, Pardo T, Cerda J, Reyes B, Holmgren NL. Prevalence of habitual snoring and associated neurocognitive consequences among Chilean school aged children. Int J Pediatr Otorhinolaryngol. 2012;76(9):1327-31.

3. O'Brien LM, Mervis CB, Holbrook CR, Bruner JL, Klaus CJ, Rutherford J, et al, Neurobehavioral implications of habitual snoring in children. Pediatrics. 2004;114(1):44-9.

4. Beebe DW. Neurobehavioral morbidity associated with disordered breathing during sleep in children: a comprehensive review. Sleep. 2006;29(9):1115-34

5. Bhattacharjee R, Kim J, Alotaibi WH, Kheirandish-Gozal L, Capdevila OS, Gozal D. Endothelial dysfunction in children without hypertension: potential contributions of obesity and obstructive sleep apnea. Chest. 2012;141(3):682-91.

6. Marcus CL, Brooks LJ, Draper KA, Gozal D, Halbower AC, Jones J, et al. Diagnosis and management of childhood obstructive sleep apnea syndrome. Pediatrics. 2012;130(3):576-84.

7. Tan HL, Kheirandish-Gozal L, Gozal D. Pediatric home sleep apnea testing: slowly getting there! Chest. 2015;148(6):1382-95.

8. Katz SL, Witmans M, Barrowman N, Hoey L, Su S, Reddy D, et al. Paediatric sleep resources in Canada: the scope of the problem. Paediatr Child Health. 2014;19(7):367-72

9. Hang LW, Wang HL, Chen JH, Hsu JC, Lin HH, Chung WS, et al. Validation of overnight oximetry to diagnose patients with moderate to severe obstructive sleep apnea. BMC Pulm Med. 2015;15:24.

10. Kirk VG, Bohn SG, Flemons WW, Remmers JE. Comparison of home oximetry monitoring with laboratory polysomnography in children. Chest. 2003; 124(5):1702-8

11. Kirk VG, Baughn J, D'Andrea L, Friedman N, Galion A, Garetz S, et al. American Academy of sleep medicine position paper for the use of home sleep apnea test for the diagnosis of OSA in children. J Clin Sleep Med. 2017;13(10):1199-203.

12. Horwood L, Brouillette RT, McGregor CD, Manoukian JJ, Constantin E. Testing for pediatric obstructive sleep apnea when health care resources are rationed. JAMA Otolaryngol Head Neck Surg. 2014; 140(7):616-23.

13. Lau A, Ewing C, Gnanapragasam J, Majaesic C, MacLean J, Mandhane PJ. Changes to a pediatric sleep disordered breathing clinic improve wait-times and clinic efficiency. Pediatr Pulmonol. 2016;51(11):1234-41.

14. Pavone M, Ullmann N, Verrillo E, De Vincentiis G, Sitzia E, Cutrera R. At-home pulse oximetry in children undergoing adenotonsillectomy for obstructive sleep apnea. Eur J Pediatr. 2017;176(4):493-9.

15. Sharma SD, Kanona H, Kumar G, Kotecha B. Latest trends in the assessment and management of paediatric snoring and sleep apnea. J Laryngol Otol. 2016;130(5):482-9.

16. Roland PS, Rosenfeld RM, Brooks LJ, Friedman NR, Jones J, Kim TW, et al. Clinical practice guidelines: Polysomnography for sleep-disordered breathing prior to tonsillectomy in children. Otolaryngol Head Neck Surg. 2011;145(1 Suppl):S1-15

17. Nixon GM, Kermack AS, Davis GM, Manoukian JJ, Brown KA, Brouillette RT. Planning adenotonsillectomy in children with obstructive sleep apnea: the role of overnight oximetry. Pediatrics. 2004;113(1 Pt 1):e19-25.

18. Amoils M, Chang KW, Saynina O, Wise PH, Honkanen A. Postoperative complications in pediatric tonsillectomy and adenoidectomy in ambulatory vs inpatient settings. JAMA Otolaryngol Head Neck Surg 2016;142(4):344-50.

\section{Publisher's Note}

Springer Nature remains neutral with regard to jurisdictional claims in published maps and institutional affiliations.
Ready to submit your research? Choose BMC and benefit from:

- fast, convenient online submission

- thorough peer review by experienced researchers in your field

- rapid publication on acceptance

- support for research data, including large and complex data types

- gold Open Access which fosters wider collaboration and increased citations

- maximum visibility for your research: over $100 \mathrm{M}$ website views per year

At $\mathrm{BMC}$, research is always in progress.

Learn more biomedcentral.com/submissions 